ARTICLE

\title{
Capturing transient antibody conformations with DNA origami epitopes
}

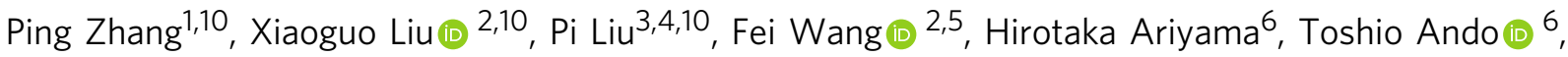 \\ Jianping $\mathrm{Lin}^{3,4}$, Lihua Wang7,8, Jun $\mathrm{Hu}^{1,7 凶}$, Bin $\mathrm{Li}^{1,7 凶} \&$ Chunhai Fan (1) $2,9 凶$
}

Revealing antibody-antigen interactions at the single-molecule level will deepen our understanding of immunology. However, structural determination under crystal or cryogenic conditions does not provide temporal resolution for resolving transient, physiologically or pathologically relevant functional antibody-antigen complexes. Here, we develop a triangular DNA origami framework with site-specifically anchored and spatially organized artificial epitopes to capture transient conformations of immunoglobulin Gs (IgGs) at room temperature. The DNA origami epitopes (DOEs) allows programmed spatial distribution of epitope spikes, which enables direct imaging of functional complexes with atomic force microscopy (AFM). We establish the critical dependence of the IgG avidity on the lateral distance of epitopes within 3-20 nm at the single-molecule level. High-speed AFM imaging of transient conformations further provides structural and dynamic evidence for the IgG avidity from monovalent to bivalent in a single event, which sheds light on various applications including virus neutralization, diagnostic detection and cancer immunotherapy.

\footnotetext{
${ }^{1}$ CAS Key Laboratory of Interfacial Physics and Technology, Shanghai Institute of Applied Physics, Chinese Academy of Sciences, University of Chinese Academy of Sciences, Shanghai 201800, China. ${ }^{2}$ School of Chemistry and Chemical Engineering, Frontiers Science Center for Transformative Molecules, Institute of Translational Medicine, Shanghai 200240, China. ${ }^{3}$ State Key Laboratory of Medicinal Chemical Biology, College of Pharmacy and Tianjin Key Laboratory of Molecular Drug Research, Nankai University, Haihe Education Park, 38 Tongyan Road, Tianjin 300353, China. ${ }^{4}$ Biodesign Center, Tianjin Institute of Industrial Biotechnology, Chinese Academy of Sciences, Tianjin 300308, China. ${ }^{5}$ Joint Research Center for Precision Medicine, Shanghai Jiao Tong University Affiliated Sixth People's Hospital South Campus, Southern Medical University Affiliated Fengxian Hospital, Shanghai 201499 , China. 6 Nano Life Science Institute (WPI NanoLSI), Kanazawa University, Kakuma-machi, Kanazawa 920-1192, Japan. ${ }^{7}$ Shanghai Synchrotron Radiation Center, Shanghai Advanced Research Institute, Chinese Academy of Sciences, Shanghai 201210, China. ${ }^{8}$ Shanghai Key Laboratory of Green Chemistry and Chemical Processes, School of Chemistry and Molecular Engineering, East China Normal University, 500 Dongchuan Road, Shanghai 200241, China. ${ }^{9}$ Institute of Molecular Medicine, Shanghai Key Laboratory for Nucleic Acids Chemistry and Nanomedicine, Renji Hospital, School of Medicine, Shanghai Jiao Tong University, Shanghai 200127, China. ${ }^{10}$ These authors contributed equally: Ping Zhang, Xiaoguo Liu, Pi Liu. ${ }^{凶}$ email: hujun@sinap.ac.cn; libin@sinap.ac.cn; fanchunhai@sjtu.edu.cn
} 
A ntibody $(\mathrm{Ab})$-antigen $(\mathrm{Ag})$ interactions are important natural defense strategies in the mammalian immune system. Their unparalleled specificity has been popularly exploited for developing tools in clinical diagnosis, treatment, and prevention, as exemplified by conventional vaccine prevention and rapidly emerging cancer immunotherapy ${ }^{1-7}$. The fundamental mechanism of $\mathrm{Ab}-\mathrm{Ag}$-binding processes is of great importance for understanding immunology, however, revealing dynamic $\mathrm{Ab}-\mathrm{Ag}$ interactions at the single-molecule level remains difficult ${ }^{8}$. Historically, the "Lock-and-Key" theory was proposed to explain the intrinsic capability of Abs to bind their cognate Ags with high specificity. Later on, the "induced-fit" model was evolved to describe $\mathrm{Ab}-\mathrm{Ag}$ interactions by taking consideration of transient conformational changes of Abs/Ags ${ }^{9,10}$. As a powerful method to determine protein structures with the atomistic resolution, X-ray crystallography provides a route to experimentally examine modes of $\mathrm{Ab}-\mathrm{Ag}$ interactions ${ }^{11,12}$. Cryogenic electron microscopy (cryo-EM) serves as a tool for imaging individual Abs at atomic resolution, offering unprecedented capability to determine complex structures. But important information on conformational flexibility and intermediate $\mathrm{Ab}-\mathrm{Ag}$ complexes are likely lost due to the lack of temporal resolution ability of cryo-EM ${ }^{13,14}$.

Atomic force microscopy (AFM) has allowed the imaging of biomolecules under physiological conditions ${ }^{15-19}$, which provides an alternative route to probe $\mathrm{Ab}-\mathrm{Ag}$ interactions ${ }^{20-23}$. Previous studies have demonstrated the potential of AFM for imaging Abs with near-atomistic resolution ${ }^{24-26}$ and its ability for quantitatively mapping nanomechanical forces of $\mathrm{Ab}-\mathrm{Ag}$ interactions at the single-molecule level ${ }^{27-29}$. Recently, high-speed (HS) AFM offers up to video-rate temporal resolution, revealing certain types of dynamic processes of IgGs, e.g., walking on the viral surface, oligomerization, and complement activation upon antigen recognition $24,30,31$. These exciting advances in unveiling distinctive molecular events of Abs reveal its compelling implications in resolving heterogeneous conformations of $\mathrm{Ab}-\mathrm{Ag}$ complexes. Nevertheless, the spatial resolution is generally sacrificed in the HS mode. High-resolution imaging of transient functional $\mathrm{Ab}-\mathrm{Ag}$ complexes with AFM is difficult to implement.

Here, we reason that transient binding conformations of a single IgG can be observed in aqueous solution at room temperature. We devise DNA origami epitopes (DOEs) to elucidate the transient binding conformations of immunoglobulin Gs (IgGs, $150 \mathrm{kDa}$ ). Using AFM, HS-AFM and single-molecule FRET (smFRET), we interrogate the structure, avidity, and dynamic binding processes of IgGs at the single-molecule level.

\section{Results}

Design and fabrication of DOEs for IgG capture. Epitope spikes often distribute unevenly on the surface of viral particles (Fig. 1a), which has been known to influence the avidity of antibodies ${ }^{32,33}$. We were inspired to design DNA origami ${ }^{34-44}$-based DOEs mimicking the distance distribution of viral epitopes to program the antibody-binding ability (Fig. 1b). To do that, six pairs of digoxin molecule $(780 \mathrm{Da})$ were site specifically anchored on the prescribed positions of a triangular DNA origami. The lateral distances of each epitope pair were separated by 3-20 nm (Supplementary Figs. 1 and 2).

Having established the DOE platform, we performed IgG capture which was imaged with AFM. DOEs were deposited onto a freshly cleaved mica substrate, which was then incubated with IgGs. Figure 1c shows a solution-phase AFM image of IgG-bound DOEs. We observed Y-shaped bright dots representing IgGs with various binding structures located on all prescribed sites of DOEs. Interestingly, on sites with the epitope distances of 8,10 , and
$16 \mathrm{~nm}$, the three arms of IgGs were clearly visible; however, the three-arm structures of IgGs were not well defined on sites with the epitope distances of 3, 5, and $20 \mathrm{~nm}$ (Fig. 1d), exhibiting epitope distance-dependent binding behaviors. Especially, the image of an IgG was blurred for the lateral distance of $20 \mathrm{~nm}$, suggesting that the bound IgG was mobile. In this situation, the lateral distance exceeds the contour length of the two arms, which possibly only supports monovalent binding of IgGs.

The high resolution of IgGs probably arises from the firm binding of DOEs, which restricts the motions of IgGs when they bivalently bind to the epitope pair (avidity of 2). Importantly, the stiff DOEs not only rigidify IgGs but prevent their random adsorption on mica. The near lying-flat orientation of IgGs provides a precise tool for measuring the size and conformational variations at different binding states at the single-molecule level. The Y-shaped structure and the size of the three arms of IgGs measured to be most $4.0 \times 4.0 \times 5.5 \mathrm{~nm}^{3}$ (Supplementary Fig. 3) fit well with the molecular structure of IgGs obtained from the Protein Data Bank (PDB entry, 1HZH) and the topographical AFM image of IgGs from electron tomography (ET) and $\mathrm{AFM}^{11,26,45}$. We assumed the two bound arms were the Fab domains where the unbound one was the Fc domain (Fig. 1e). We assigned the bound two arms as Fab regions. We also note that the hinge domain was visible, as shown in Fig. 1e.

Conformational flexibility of DOE-confined IgGs. Having established the DOE capture system, we next explored the conformational flexibility of DOE-confined IgGs with HS-AFM. Figure 2a shows HS-AFM snapshots ( 2 frame $\mathrm{s}^{-1}$ ) of IgGs bound to several DOEs sites $(5,8,10$, and $16 \mathrm{~nm})$. We observed that IgGs confined in all the four separation distances showed wagging motions, as indicated by the arrows in Fig. 2a. Generally, two types of wagging motions exist: out-of-plane wagging (the $5 \mathrm{~nm}$ site, white circles) and in-plane wagging $(8,10$, and $16 \mathrm{~nm}$ sites, white arrows). The former appears to be a whole-body motion when the two Fab domains are held tight, whereas the latter is primarily the motion of the Fc domain (Supplementary Figs. 4 and 5). We also note that this flexibility of binding conformations of IgGs is likely of relevant physiological functions ${ }^{46-51}$.

Next, we examined the relationship between the measured distance of Fab barycenters and the epitope distances (Fig. 2b). Despite the dynamic motions of IgGs, we found that the distance of Fab barycenters were nearly fixed, providing a precise measure for the stretching of Fab domains. As a general trend, the measured distances increased along with the designed epitope distances, suggesting that the two Fab domains of a single IgG could be programmably stretched using epitope distanceengineered DOEs. Of note, we found the deviation of two distances especially at the longer distances. The measured distances of two Fabs in IgGs are 4.6 $\pm 1.0,6.0 \pm 1.0,8.1 \pm 1.0$, $9.7 \pm 0.8$, and $11.3 \pm 1.2 \mathrm{~nm}$ for the designed digoxin distances 3 , $5,8,10$, and $16 \mathrm{~nm}$, respectively $(n=10$, central values $=$ average values, and $\pm=$ standard deviations (SD)) (Fig. 2c, Supplementary Fig. 6). This is possibly because the measured distance is the distance between the barycenters of two Fabs, which is smaller than the epitope distances, especially when the two Fabs are stretched (e.g. at an epitope distance of $16 \mathrm{~nm}$ ). Overall, we showed that DOEs could program the stretching states of IgGs, and image the stretching conformations at room temperature.

Dynamics of DOE-IgG binding. To probe the DOE-IgG-binding dynamics, we employed HS-AFM ( 2 frames $\left.\mathrm{s}^{-1}\right)$ to trace transitional binding of IgGs on tens of DOEs (Fig. 3a, Supplementary Fig. 7). We observed a three-stage DOE-IgG-binding process: (i) wandering state, (ii) monovalent binding, and (iii) bivalent 
a

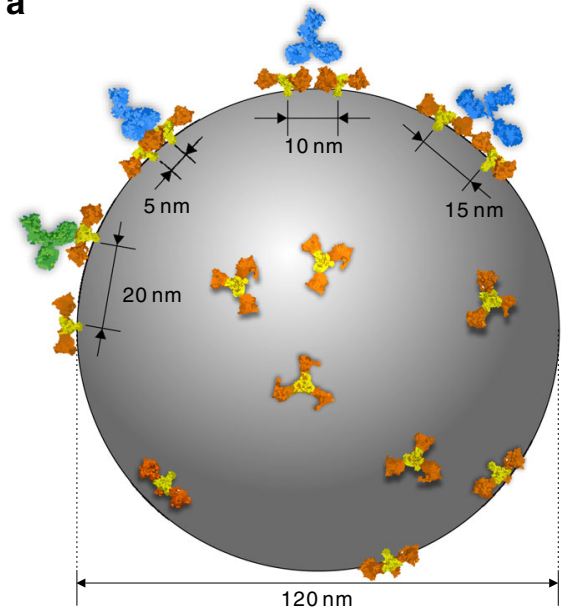

b

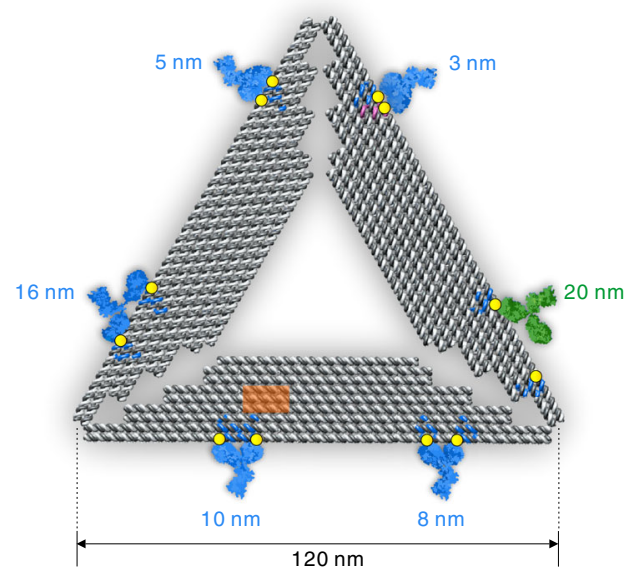

c

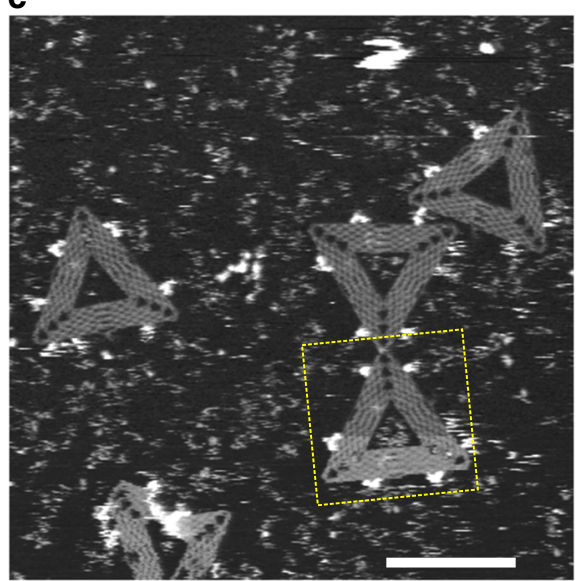

d

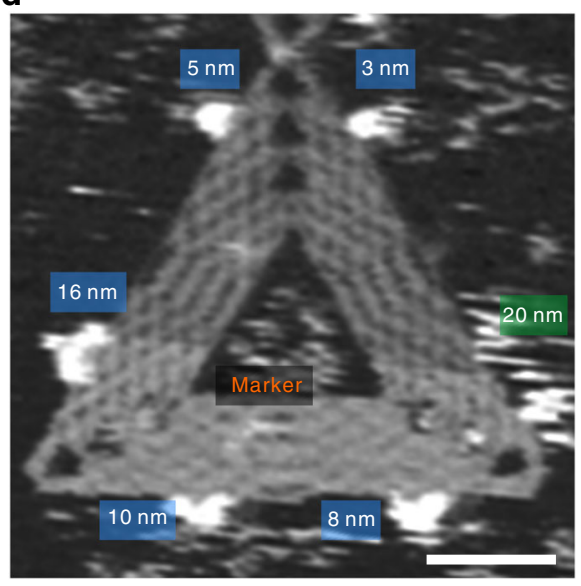

e
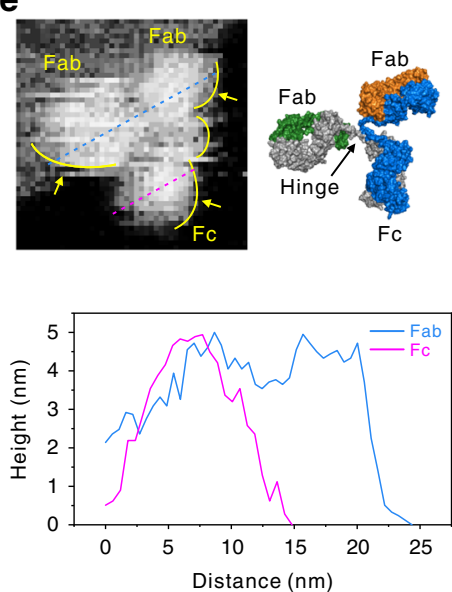

Fig. 1 DOE-based capture of IgGs. a Schematic representation of non-even distribution of epitope spikes on the surface of a viral particle. $\mathbf{b}$ Designed, virus-mimicking DOEs for lgG capture and binding. Artificial epitope (digoxin, yellow), DNA origami (gray), modified staple DNA strands (blue and pink), IgGs (bivalent binding, blue; monovalent binding, green). c PeakForce-AFM image for DOE-based capture of IgGs. Scale bar, $100 \mathrm{~nm}$. $\mathbf{d}$ Enlarged view of the dashed yellow square in panel c. Scale bar, $20 \mathrm{~nm}$. e A high-resolution HS-AFM image of an Fc and two Fab domains of the bound lgG (upper left, yellow arrows); atomic structure of IgG (Protein Data Bank (PDB) entry $1 \mathrm{HZH}$, upper right); cross-sectional profile of three domains (Fab, blue and Fc, magenta) in a DOE-bound IgG (bottom). Source data are provided as a Source Data file.

binding state (Fig. 3a). Figure 3b shows three exemplary binding events for the 3,10 , and $16 \mathrm{~nm}$ sites on DOEs. Especially, for $10 \mathrm{~nm}$ site (middle raw), initially, we found the IgG molecule wandering in the bulk solution out of plane until $2.0 \mathrm{~s}$ (highlighted by orange circle). Then, in $<0.5 \mathrm{~s}$, one Fab arm of the IgG was captured by an epitope spike (highlighted by cyan circle). Finally, within another $0.5 \mathrm{~s}$, the other Fab arm was captured by the proximal epitope spike. Then the two Fab arms were firmly held by the epitope pair, leaving a wagging Fc domain (highlighted by green circles). The phenomena that IgG underwent three typical conformational states before achieving bivalent binding were observed in various designed sites, except on the $20 \mathrm{~nm}$ site. It should be noted that $10 \mathrm{~nm}$ sites had the shortest monovalent-to-bivalent binding intervals of $\sim 1.0 \mathrm{~s} \quad(n=7$, $\mathrm{SD}=0.5 \mathrm{~s}$ ) (Supplementary Table 7, Supplementary Fig. 8). For other distances, the intervals were generally $\sim 85.0 \mathrm{~s}(3 \mathrm{~nm}, n=5$, $\mathrm{SD}=46.0 \mathrm{~s}), \sim 49.0 \mathrm{~s}(5 \mathrm{~nm}, n=9, \mathrm{SD}=31.0 \mathrm{~s}), \sim 3.0 \mathrm{~s}(8 \mathrm{~nm}$, $n=5, \mathrm{SD}=2.4 \mathrm{~s})$, and $23.0 \mathrm{~s}(16 \mathrm{~nm}, n=3, \mathrm{SD}=4.4 \mathrm{~s})$, respectively (Supplementary Table 7, Supplementary Figs. 10, 12, 14, and 16). In addition, the dynamic motions of the Fab arms are illustrated in detail with magnified HS-AFM images and movies (Supplementary Figs. 9, 11, 13, 15, and 17, Movies 1-5).
Next, we employed total internal reflection fluorescence (TIRF) microscopy to perform smFRET to validate the three-stage DOEIgG binding mechanism. To this end, Alexa 647 acceptor was labeled on the IgG, a pair of ATTO 550 donors was located proximal to the epitope spikes $(10 \mathrm{~nm})$ on DOE. Figure $3 \mathrm{c}$ shows successive step-down fluorescence trajectory in a typical DOEIgG-binding event. Given that photobleaching of the two ATTO 550 dyes generated only two descending steps with intervals generally longer than $10 \mathrm{~s}$ (Fig. 3d, Supplementary Fig. 18), the initial fluorescence intensity descending steps at $\sim 7 \mathrm{~s}$ and $\sim 10 \mathrm{~s}$ were assigned to a two-step FRET change brought by IgG monovalent and bivalent binding states (Fig. 3c, insertions). Generally, the smFRET analysis complements HS-AFM to establish the three-stage DOE-IgG binding mechanism (Supplementary Figs. 18 and 19).

Engineering DOEs for evoking the IgG avidity. To quantify the epitope distance effect on the IgG avidity, we explored the transition binding kinetics of IgGs from monovalent (avidity of 1) to bivalent (avidity of 2) using HS-AFM. The transition time varied greatly from sub-second to over 100 second at different epitope distance. We found that the $10 \mathrm{~nm}$ site led to the shortest 
a
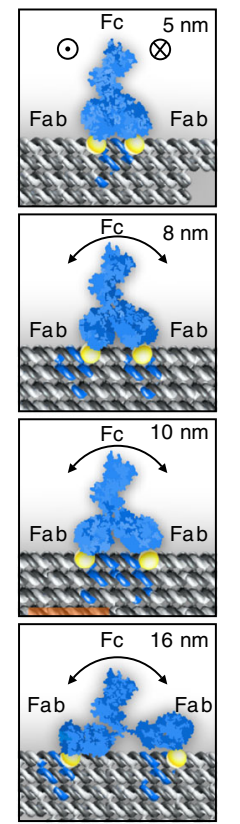
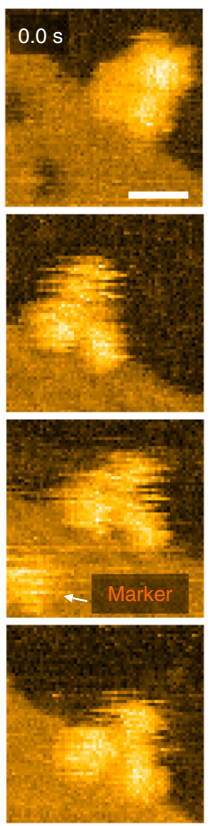
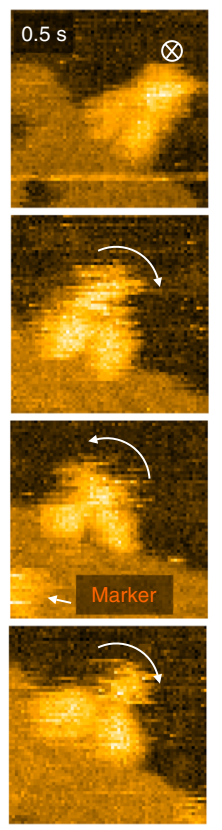
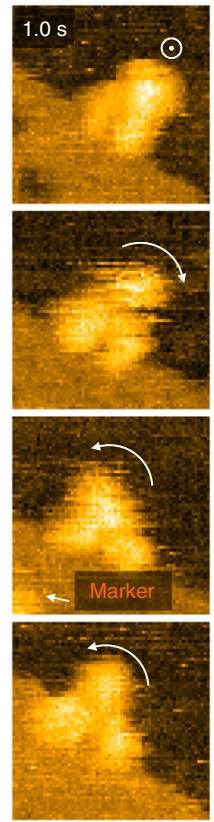

b

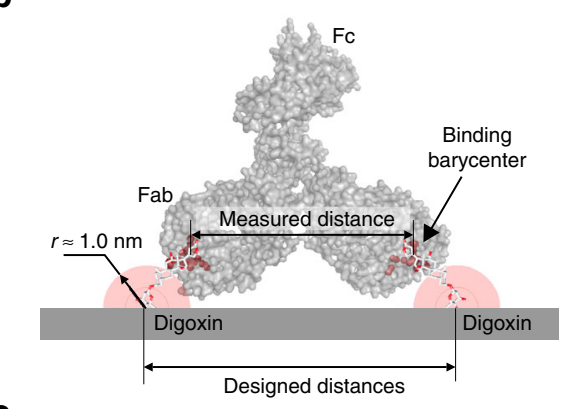

C

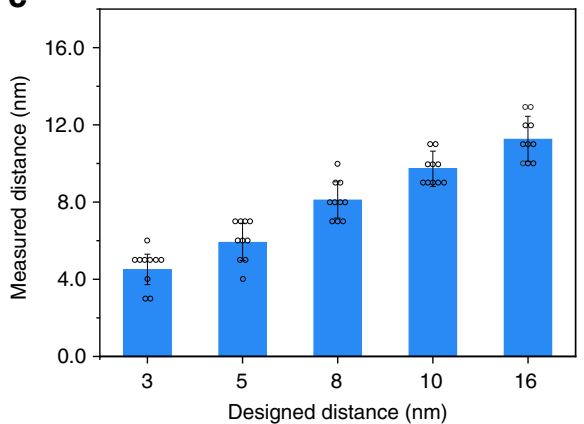

Fig. 2 HS-AFM characterization of the conformational flexibility and measured Fab-Fab distance of DOE-confined IgGs. a Schematics representing the distinct conformations of single lgGs captured by DOEs with designed distances of 5, 8, 10, and $16 \mathrm{~nm}$, respectively (left). Snapshot HS-AFM images (2 fps) of single IgGs bound to various designed lateral distances of epitopes (right). Different conformations of lgGs, and positional fluctuations of the mass barycenters of Fc domains, are observed (right, white arrows). Scale bar, $10 \mathrm{~nm}$. b Schematic illustration of the measured distances between the barycenters of two Fab domains, and designed distances of paired epitopes (black lines). c Relationship between the designed digoxin distances (3, 5, 8, 10, and $16 \mathrm{~nm})$ and measured distances of Fabs in $\operatorname{lgGs}(4.6 \pm 1.0,6.0 \pm 1.0,8.1 \pm 1.0,9.7 \pm 0.8$, and $11.3 \pm 1.2 \mathrm{~nm})$. Central values represent average values, and error bars represent the standard deviations, as calculated from independent experiments $(n=10)$. Source data are provided as a Source Data file.

transition time (Fig. 4a, upper), implying a preferable distance for bivalent binding of IgGs. Shortened distances (3, 5, and $8 \mathrm{~nm}$ ) resulted in longer transition time, possibly due to the increased steric effects under these circumstances. Increasing the distance to $16 \mathrm{~nm}$ also slightly extended the transition, suggesting that stretching the two IgG arms requires significant relaxation time which is consistent with previous studies that, structurally, the two Fab arms cannot be stretched over $20 \mathrm{~nm}^{33}$. The epitope distance effect on the IgG avidity was further tested using PeakForce-AFM. The difference between $8 \mathrm{~nm}$ and $10 \mathrm{~nm}, 10 \mathrm{~nm}$ and $16 \mathrm{~nm}$ is statistically significant $\left({ }^{*} P<0.05\right.$, ANOVA) when judged from the monovalent/bivalent binding efficiency. Compared with monovalent/bivalent binding efficiency of $19.5 \%$ / $80.6 \%$ for distance of $10 \mathrm{~nm}$, monovalent/bivalent binding efficiency of $32.7 \% / 60.1 \%$ and $47.1 \% / 53.3 \%$ correspond to distances of 8 and $16 \mathrm{~nm}$, respectively (Supplementary Fig. 20), indicating that it is more difficult to achieve bivalent binding at both 8 and $16 \mathrm{~nm}$ as compared to $10 \mathrm{~nm}$ distance.

The bivalent binding efficiency was further evaluated with different epitope distances (Fig. 4a, bottom, Supplementary Figs. 20-24). Consistent with the transition kinetics, we found that the $10 \mathrm{~nm}$ distance led to the highest bivalent binding efficiency, whereas shorter or longer distances decreased the efficiency. The generality of this distance-dependent-binding efficiency was substantiated with the use of biotin and antibiotin IgG, as well as cholesterol and anti-cholesterol IgG pairs on designed DOEs (Supplementary Figs. 25-32). We should note that although DNA origami enables the modification of molecules with nanometer precision, entities modified on the ends of the DNA staple strand may endure uncertainty. For example, Shaw et al. ${ }^{41}$ showed that the optimal distance for IgG binding to two epitopes is $16 \mathrm{~nm}$, whereas our results showed that this distance is about $10 \mathrm{~nm}$. Different uncertainty estimation method, characterization technique, and design strategies for DNA origami that cause extensions at binding sites are presumable reasons for explaining this discrepancy (Supplementary Fig. 2).

To understand the mechanism of the epitope distance-dependent avidity of IgGs, we performed coarse-grained molecular dynamics (MD) simulations on IgG-DOE binding. Interestingly, we found that IgG had the lowest potential of mean force (PMF) at a binding barycenter distance (d) of $\sim 9.4 \mathrm{~nm}$ (Fig. 4b). According to the PMF equation:

$$
\mathrm{PMF}=-k T \ln P(r),
$$

where $P(r)$ is the probability that a certain $\operatorname{IgG}$ conformation forms at a given $d, k$ is the Boltzmann constant, and $T$ is the system temperature. Hence, conformations at $\sim 9.4 \mathrm{~nm}$ have the highest probability of occurrence and the lowest potential, which is consistent with the experimental results that the $10 \mathrm{~nm}$ sites are kinetically and thermodynamically favored. The PFM profile shows that conformations with $d$ smaller or greater than $9.4 \mathrm{~nm}$ were all energetically unfavored. In other words, the favored $\sim 9.4 \mathrm{~nm}$ conformation at equilibrium corresponds to the average value of the Fab-Fab angle $\sim 105^{\circ}$. This finding is in good agreement with previous structure analysis of the intact IgGs with Fab-Fab angle $\sim 110^{\circ}$ in cryo-EM ${ }^{46}$. Note that when $d>16 \mathrm{~nm}$, the molecular structure of IgG start to collapse during computation. Taking the active radius into consideration, the maximum stretching length of IgGs is no more than $\sim 18 \mathrm{~nm}$, which is consistent with our experimental observations.

We further employed MD simulations to compute the dynamic conformations of bivalently bound IgGs (Fig. 4c). 

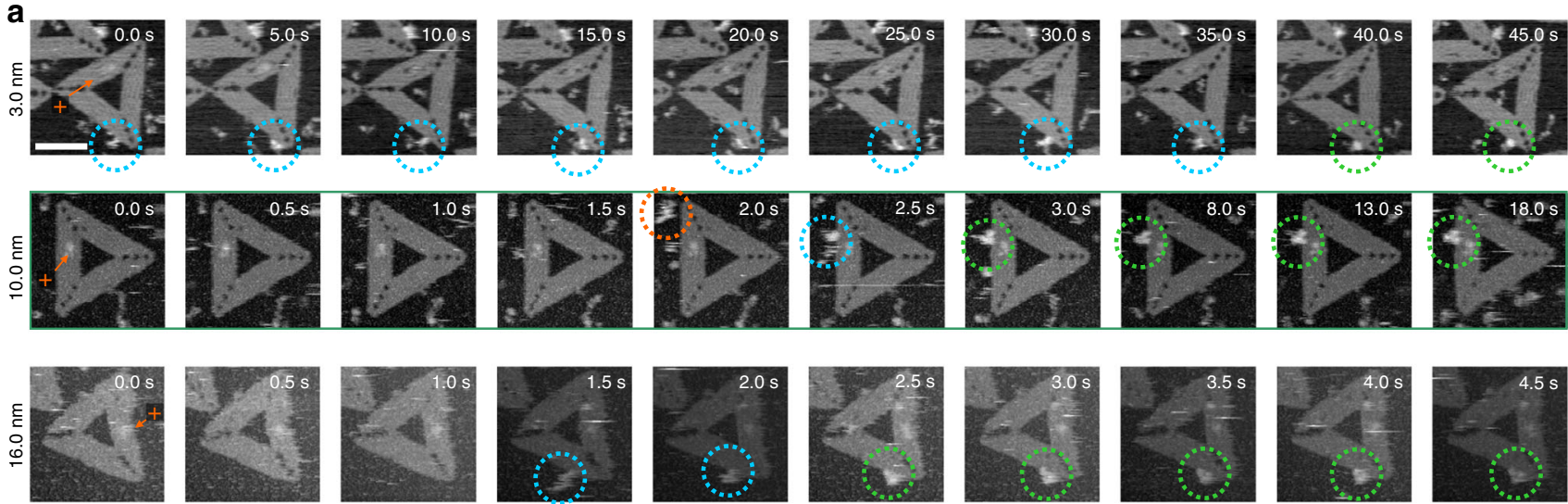

b
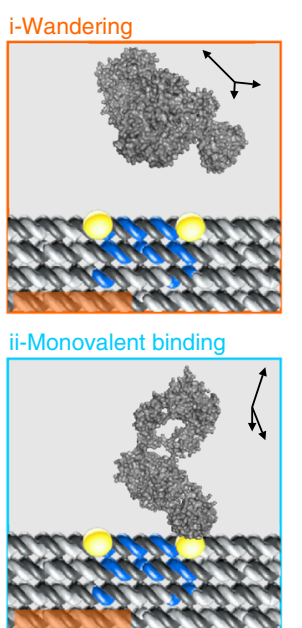

iii-Bivalent binding

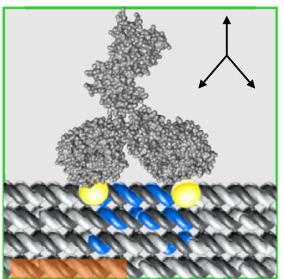

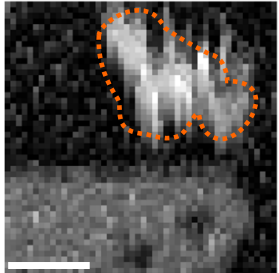
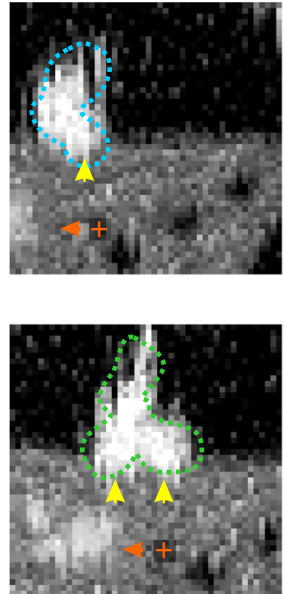

c

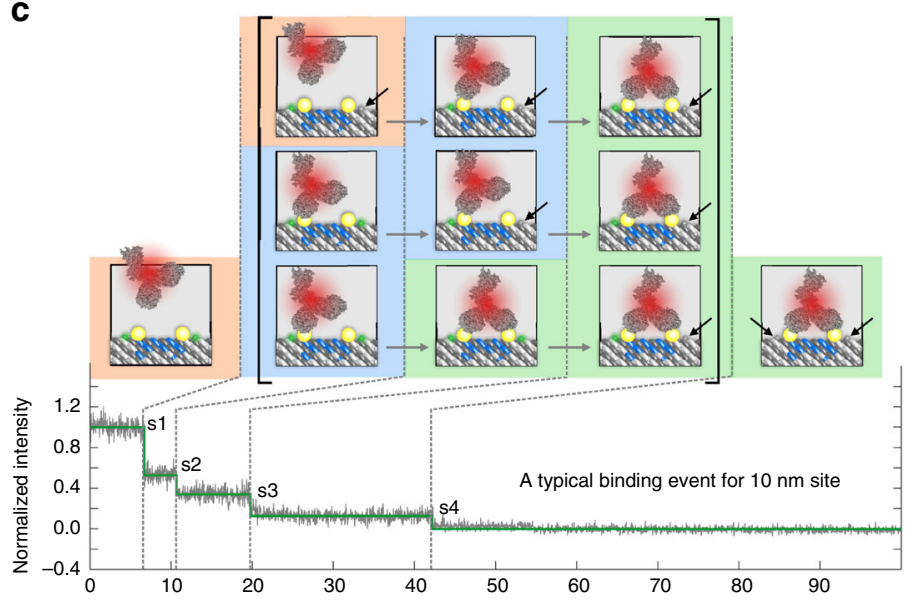

d

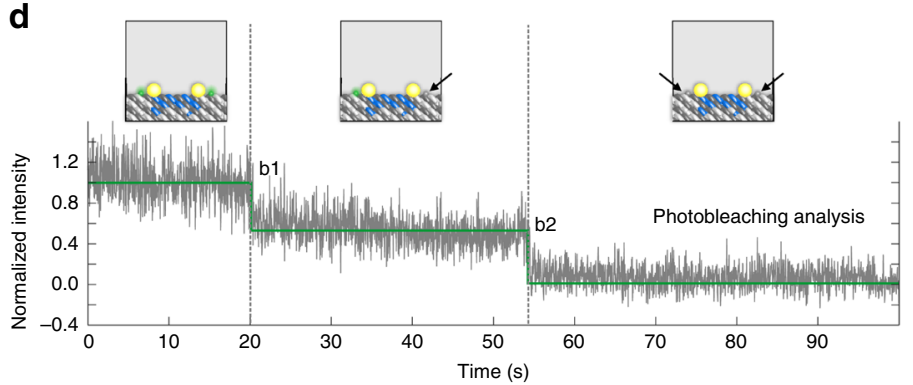

Fig. 3 Dynamics of IgG binding to DOEs. a HS-AFM images for temporal evolution of the three-stage binding process, from wandering (orange circle) to monovalent binding (cyan circle), then bivalent binding (green circle) states for DOEs with designed distances of 3, 10, and 16 nm, respectively. Scale bar, $60 \mathrm{~nm}$. b Schematic of the three-stage binding process and corresponding HS-AFM images (10-nm site: representative images of over ten independent replicates). The black arrows within each coordinate indicate the orientation of the lgG model. The dashed contours highlight the observed lgGs at different stages. The yellow arrows indicate the binding sites. The orange arrows indicate the maker, and orange plus signs represent positive orientation of DOE platform. Scale bar, $10 \mathrm{~nm}$. c Exemplary single-molecule FRET trajectory (gray, raw $31.25 \mathrm{~Hz}$ data), and hidden Markov model fit (green) of a binding event for $10 \mathrm{~nm}$ site. A pair of ATTO 550 dye (donor, green dots) is labeled to the proximity of the epitopes. Alexa 647 dye (acceptor, crimson dots) is labeled on IgG. Note that two photobleaching events are inserted in the three-stage binding process observed by HS-AFM, resulted in a total five fluorescence intensity levels. The second bleaching could only occur at s4, while the first bleaching may occur at s1, s2, or s3. d Photobleaching analysis of the DOEs only system showing two-step (b1 and b2) signal drop due to the successive bleaching of two ATTO 550 dyes. Source data are provided as a Source Data file.

We found that IgG could take conformations ranging from highly compact $(d=6.2 \mathrm{~nm})$ to far stretched $(d=16.0 \mathrm{~nm})$. The design of epitope distances on DOEs provides a straightforward and programmable approach to observe these transient, functional conformations at room temperature. Of note, the measured barycenter distance $d$ is smaller than the designed distance D. Importantly, we imaged these transient conformations of IgGs in solution with AFM, which were all captured at prescribed distances (Fig. 4d).

\section{Discussion}

In this study, we devised DOEs to mimic the distance distribution of epitopes on viral particles by exploiting the spatial addressability of DNA origami. The positioning ability and stiffness of DOEs enables room-temperature freezing of IgGs for high-resolution imaging of transient, functional IgG binding conformations at the single-molecule level. The precision in programmable control of the lateral distances of epitopes on DOEs allows unequivocal determination of the epitope 
a

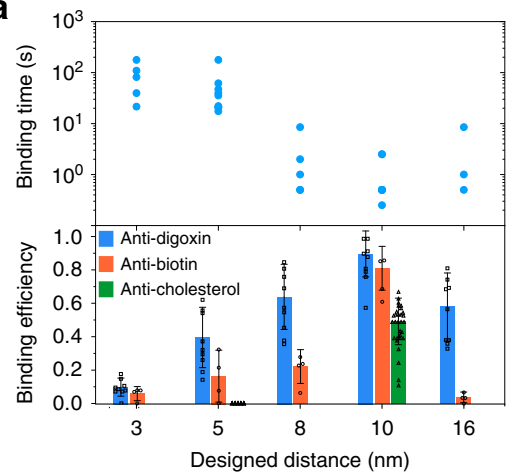

C
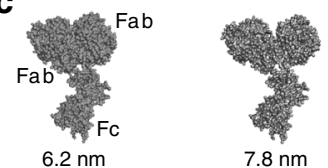

d

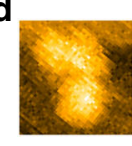
$D=5 \mathrm{~nm}$
$d=4.8 \mathrm{~nm}$

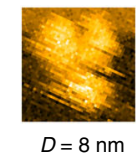

$D=8 \mathrm{~nm}$
$d=7.5 \mathrm{~nm}$
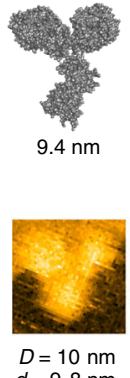

b
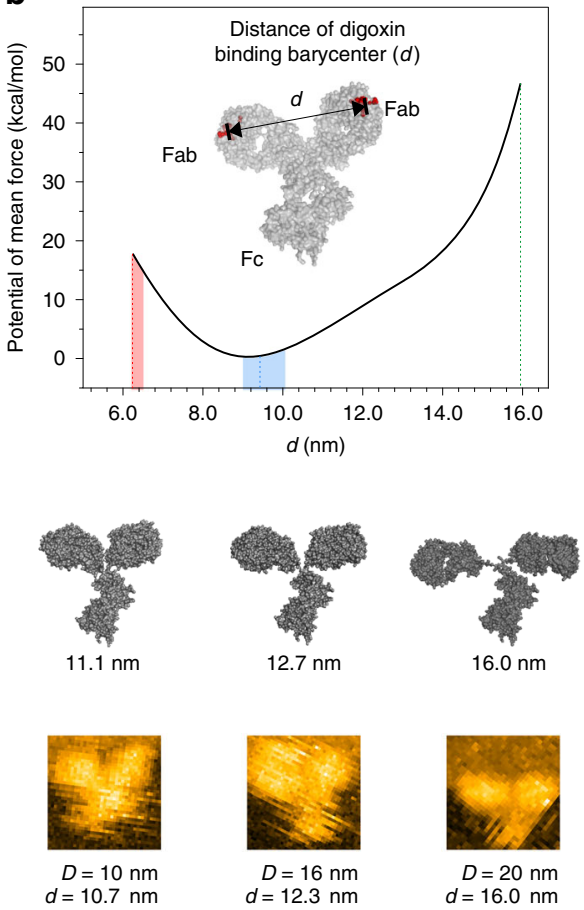

Fig. 4 Engineering DOEs for evoking the IgG avidity. a Scatter plot for the monovalent-to-bivalent-binding transition kinetics of IgG-DOE (upper, HSAFM), and histogram of IgG binding efficiency as a function of the designed distance on DOEs (bottom, PeakForce-AFM). The error bars represent the standard deviation, calculated from three independent experiments for each type of lgG. For the anti-digoxin, anti-biotin, and anti-cholesterol lgG types, the numbers of DNA origami used for each DOE were 252, 372, and 661, respectively. b Course-grained molecular dynamics calculations. Calculated energy diagram for IgG binding between mass barycenters at various distances. The most preferred binding site distance of IgGs was $9.5 \mathrm{~nm}$. Conformations with binding site distance short or longer than $9.5 \mathrm{~nm}$ are all energy unfavorable. Especially, when distance longer than $15.0 \mathrm{~nm}$, the potential of mean force was dramatically increased. It also implies that conformations with or close to $9.5 \mathrm{~nm}$ has the largest probability of occurrence. c, $\mathbf{d}$ Typical simulated free IgG conformations using course-grained molecular dynamics calculations and corresponding HS-AFM images for the bivalently bound lgGs on DOEs. Source data are provided as a Source Data file.

distance-dependent IgG avidity. This DOE platform also supports HS-AFM and smFRET analysis to probe the dynamics of single IgG binding events on DOEs.

The dynamics of $\mathrm{Ab}-\mathrm{Ag}$ binding has been extensively studied theoretically and experimentally $24,52-56$. However, direct capture of transient binding conformations of $\mathrm{Ab}-\mathrm{Ag}$ complexes at room temperature remains difficult. Our DOE platform provides direct structural evidence for the transient, functional conformations of IgGs at room temperature, which may deepen our understanding of physiologically or pathologically relevant $\mathrm{Ab}-\mathrm{Ag}$ complexes. Especially, we find that IgGs can take flexible conformations ranging from high compact to far stretched in response to shortto-long epitope distances. Importantly, the binding kinetics and efficiency for bivalent IgG binding is the highest when the two epitopes are separated by $\sim 10 \mathrm{~nm}$.

The distance-dependent binding of IgGs on epitopes has important physiologically or pathologically relevance. Viruses have been well known to adopt wise strategies to escape Abmediated neutralization strategy by tuning the spatial distribution of epitope spike on their surfaces ${ }^{32}$, e.g. average spike distances on the surface of HIV is $>20 \mathrm{~nm}$, which is far beyond the span of two Fabs in a single $\mathrm{Ab}$ molecule ${ }^{33}$. Consequently, the flexibility of IgG binding with the two arms is critically important for their affinity/avidity ${ }^{51}$. The designability and programmability of DOEs thus offer an intuitive method to imitate viral epitope distribution. DOEs thus not only increase the design space for understanding $\mathrm{Ab}-\mathrm{Ab}$ interactions at the single-molecule level but also provide a potentially powerful platform for engineering immunological tools.

\section{Methods}

Materials. The long single-stranded M13mp18 DNA molecule was obtained from New England Biolabs (NEB). Digoxin-labeled, biotin-labeled, cholesterol-labeled, cholesterol-modified poly A (chol-A), and the rest of staple strands were bought from Sangon Biotech Co., Ltd (Shanghai, China). ATTO 550-labeled DNA short staple strands were bought from Takara, China. Anti-digoxin antibody and antibiotin antibody were obtained from Sigma Aldrich, China. Anti-cholesterol antibody was brought from Lifespan Bioscience, Inc. 1,2-Dioleoyl-sn-glycero-3-phosphocholine (DOPC) and 1,2-dimyristoyl-sn-glycero-3-phosphocholine (DMPC) were obtained from Avanti Polar Lipids, USA. Alexa Fluor 647-IgG (Alexa 647$\mathrm{IgG})$ was obtained from the Jackson laboratory, USA.

All reagents were kept at $-20^{\circ} \mathrm{C}$ until use. Deionized (DI) Water used in the experiments was $18.2 \mathrm{M} \Omega \mathrm{cm}^{-1}$. Milli-Q water was generated by a Millipore system. Polycarbonate (PC) membrane (Whatman, Fisher Scientific) with a pore diameter of $100 \mathrm{~nm}$ was used in vesicle extrusion. Millipore was obtained from GE Healthcare (Little Chalfont, UK). The PCR instrument used was the Eppendorf Mastercycler Personal Machine. The concentration of DNA origami was measured with NanoPhotomer-P330 (IMPLEN, Munich, Germany).

Design and fabrication of DOEs for IgG capture. The DOE comprised a triangular DNA origami tile fixed with epitopes. The sites and sequences of the staple strands for generating the DNA origami have been previously reported ${ }^{34}$. The DOEs were based on paired distances of digoxins, biotins, or cholesterols on the DNA origami. To obtain potentially accurate distance for the paired epitopes, all epitopes were modified directly at the $5^{\prime}-, 3^{\prime}$ - and/or interval of the staple strand on the DNA origami, and no extra linker was added. The designed distances of the paired epitopes (approximately $3,5,8,10,16$, and $20 \mathrm{~nm}$ ) were based on the numbers of nucleotides within the DNA origami: a one-turn helix (32 nt) had a length of $10.6 \mathrm{~nm}$. However, some uncertainties can be expected due to the flexibility of the helix structure, the size of epitope, and considering that the B-helix structure has a distance of $0.34 \mathrm{~nm}$ per base pair and a $2.0 \mathrm{~nm}$ diameter per helix, we estimated that the spatial variance of the designed distance was $<3.0 \mathrm{~nm}$. Therefore, all lateral distances are described in terms of their design distances, unless mentioned. 
The sites and sequences of (1) the staple strands labeled with digoxin, biotin, cholesterol, and ATTO 550; (2) the extended sequences of staple strands for anchoring DNA origami on a supported lipid bilayer (SLB); and (3) the DNA origami makers used are listed as Supplementary Tables 1-6, respectively. All staple sequences listed in the tables are presented in $5^{\prime}-3^{\prime}$ order.

Constructing a DOE based on assembly with digoxin, or biotin, or cholesterol using DNA origamis. The DOE was constructed by assembly of triangular DNA origami tile, with digoxin/biotin/cholesterol-labeled replacing strands. For smFRET experiments, strands labeled with ATTO 550 and extended by 20 -dT were added during the self-assembly process. Self-assembly was conducted in TAE/ $\mathrm{Mg}^{2+}$ buffer ( $40.0 \mathrm{mM}$ Tris, $2.0 \mathrm{mM}$ EDTA, and $\left.12.5 \mathrm{mM} \mathrm{MgCl}_{2}, \mathrm{pH} 8.0\right)^{57}$. Briefly, a $5.0 \mathrm{nM}$ single-stranded M13mp18 DNA, a 10-fold molar excess of staple strands, and 10 -fold molar excess of digoxin, biotin, or cholesterol-labeled staple strands were mixed, followed by annealing treatment from $95^{\circ} \mathrm{C}$ to $4{ }^{\circ} \mathrm{C}$ at a speed of $0.1^{\circ} \mathrm{C}$ per $10 \mathrm{~s}$. The excess staple strands were removed by ultrafiltration three times using $1 \times \mathrm{TAE} / \mathrm{Mg}^{2+}$ buffer using $100 \mathrm{kDa}$ cutoff filters (Amicon).

For preparing the DOEs used in the single-molecule smFRET experiments, single-stranded M13mp18, a 10-fold molar excess of staple strands, a 10-fold molar excess of digoxin, and a 400-fold molar excess of ATTO 550-labeled strands were mixed, followed by the same annealing and ultrafiltration process described above.

Preparation of the SLB. The small unilamellar vesicles composed of DOPC or DMPC were generated ${ }^{58}$. A particular volume of chloroform solution of DOPC or DMPC was placed in a $25 \mathrm{ml}$ round-bottomed flask. Then, the lipid film was dried under a stream of $\mathrm{N}_{2}$ using a rotary evaporator to evaporate the chloroform. The thoroughly dried mixture was immersed in DI water and sonicated for $10 \mathrm{~min}$. A lipid solution $\left(5 \mathrm{mg} \mathrm{ml}^{-1}\right)$ was extruded through a pore of polycarbonate membrane $(100 \mathrm{~nm}$ in diameter) $>30$ times. The resulting small unilamellar vesicles solution was used immediately.

An SLB was generated on a cover glass by fusion of small unilamellar vesicles. The cover glass used to support the SLB glass was dried using $\mathrm{N}_{2}$ after sonicating for $15 \mathrm{~min}$ in chloroform, acetone, ethanol, $\mathrm{KOH}$, and DI water successively to avoid influence of fluorescence background. Then, a quadrate chip fence in an edge length of $0.5 \mathrm{~cm}$ was attached to the cover glass to construct a sample chamber, using $50 \mu \mathrm{l}$ of small unilamellar vesicles solution for incubation for $30 \mathrm{~min}$ at $25^{\circ} \mathrm{C}$. Excess unfused small unilamellar vesicles were removed by thoroughly rinsing with DI water.

Tethering of the DOE on the SLB. Poly-A staple strands ( $400 \mathrm{nM})$ were added to the prepared SLB for $1 \mathrm{~h}$ incubation at $25^{\circ} \mathrm{C}$. Then, the DOE solution $(100 \mathrm{pM})$ was added for $30 \mathrm{~min}$ incubation at $25^{\circ} \mathrm{C}$, after washed by $1 \times \mathrm{TAE}$ to remove the excess poly-A staple strands. The resulting tethered DOEs on the SLB were ready to use after the excess DOEs were washed away using $1 \times$ TAE buffer.

AFM sample preparation. To quantify binding efficiency, experiments of DOEbound IgGs were conducted in solutions, in which the concentration of the DNA origami in the reaction was kept in below $0.2 \mathrm{nM}$. Anti-digoxin antibody (Catalog Number: D8156) was diluted 800 to 3200 times with TAE buffer before reaction with an equal volume DOE solution. Anti-biotin antibody (Catalog Number B7653) and anti-cholesterol antibody (Catalog Number LS-C295824) were diluted with TAE buffer into final concentration from 30 to $90 \mathrm{nM}$ and 78 to $312 \mathrm{nM}$, respectively.

HS-AFM imaging. HS-AFM Images were collected using tapping mode HS-AFM (RIBM, Japan). Silicon cantilevers (AC40, Olympus, Tokyo, Japan) with nominal spring constants of $0.09 \mathrm{~N} \mathrm{~m}^{-1}$ and a resonance frequency of $110 \mathrm{kHz}$ were used. HS-AFM was performed at room temperate $\left(\sim 25^{\circ} \mathrm{C}\right)$.

To capture the dynamic epitope-IgG binding process, $5 \mu \mathrm{l}$ of digoxin-labeled DNA origami was incubated on freshly cleaved mica for $5 \mathrm{~min}$. The sample was mounted on the AFM liquid cell filled with $30 \mu \mathrm{l} 1 \times \mathrm{TAE}-\mathrm{Mg}^{2+}$ imaging buffer. After capturing an intact DNA origami, a final diluted ratio $1: 10,000$ IgG in $1 \times$ TAE buffer was added to the liquid cell by pumping. Images were captured at a rate of $0.5 \mathrm{~s}$ with a scan area of $150 \times 150 \mathrm{pixel}^{2}$. To obtain high-resolution images of IgG conformation, images were captured at a rate of $0.1 \mathrm{~s}$ at a scan area of $70 \times 70$ pixel $^{2}$.

HS-AFM data analysis. The HS-AFM data were analyzed using kodec analysis software. The HS-AFM movies were drift corrected and contrast adjusted.

PeakForce-AFM imaging. Images were collected in PeakForce tapping mode (AFM MultimodeVIII, Veeco, Plainview, NY, USA). Cantilevers with nominal spring constants of $0.12 \mathrm{~N} \mathrm{~m}^{-1}$ (Sharp Silicon Nitride Lever (PEAKFORCE-HIRSF-B; Bruker, Billerica, MA, USA)) were applied using a typical scanning speed of $1-2 \mathrm{~Hz}$. To capture images of the three types of $\mathrm{Ab}-\mathrm{Ag}$ complexes formed in solution, DOEs were mixed with IgGs in $1 \times$ TAE buffer $(\mathrm{pH} 8.0)$. After $2 \mathrm{~h}$ incubation at $25^{\circ} \mathrm{C}$, a $3 \mu \mathrm{l}$ drop of the formed $\mathrm{Ab}-\mathrm{Ag}$ complex solution was adsorbed on a freshly cleaved mica surface for incubation for $5 \mathrm{~min}$. The resulting samples were mounted in liquid cell in $1 \times \mathrm{TAE} / \mathrm{Mg}^{2+}$ buffer for imaging. All images were flattened and analyzed using NanoScope Analysis software.

smFRET measurement. All smFRET experiments were performed using an ATTO 550-Alexa 647 coupling system in which each DNA origami was modified with one ATTO 550 molecule near one digoxin molecule using a distance of $10 \mathrm{~nm}$.

SmFRET on lipid bilayer. All smFRET experiments were performed on a commercial TIRF microscope (N-storm, Nikon) using a $\times 100$ objective lens (NA 1.49) and an electron multiplying charge-coupled devices (EMCCD) camera (iXon 3 , Andor). A solid-state laser operating at $561 \mathrm{~nm}(200 \mathrm{~mW})$ and $640 \mathrm{~nm}(200 \mathrm{~mW})$ was used to excite the fluorescence of ATTO 550 and Alexa 647, respectively. ATTO and Alexa signals were collected separately.

The reaction fluorescence signals were obtained as following: Firstly, we localized an imaging region $\left(40 \times 40 \mu \mathrm{m}^{2}\right)$ in which several static bright spots were observed using $561 \mathrm{~nm}$ laser excitation. Then, Alexa-labeled IgGs were immediately added to the sample chamber, and the fluorescence intensity of ATTO 550 was recorded with a $40 \mathrm{~ms}$ time resolution. The obtained videos were used as for subsequent signal of smFRET.

SmFRET data analysis. Image analysis was carried out using Image J software. The Bio-Formats plugin was used to extract co-localized ATTO and Alexa spots to obtain the FRET signal. Individual ATTO-Alexa pairs that dropped in successive steps revealed changes in fluorescence intensity during IgG binding. These pairs are described in Supplementary Figs. 18 and 19, which also provide the distribution of fluorescence intensity of the ATTO 550-DNA-origami after Alexa-IgG binding.

Hidden Markov Model (HMM) is widely used for identifying hidden states from noisy traces, especially in single-molecule FRET analysis. Which is specified by the following components: a set of $N$ states $\left(Q=q_{1} q_{2} \ldots q_{N}\right)$, a transition probability matrix $A$ ( $A=a_{11} \ldots a_{i j} \ldots a_{N N}$, each $a_{i j}$ representing the probability of moving from state $i$ to state $j$ ), a sequence of observations $O$, a sequence of emission probabilities $B$, an initial probability distribution over states $\pi$. State assignment was performed using open source hmmlearn package (https://pypi.org/project/hmmlearn/) in Python 3.6. Observed fluorescence intensity sequence was read in as $O$. $Q$ was initiated with a sequence of intensity values with equal intervals from minimum to maximum of observed intensity. Transition matrix $A$ was randomly generated initially. Initial probability distribution was assumed as a uniform distribution on all states.

Coarse-grain MD simulation. Initial conformation generation: The CG model of IgGs was converted from full-atom structure (pdb number: ligt) with parameters of martini CG version 2.2 (refs. ${ }^{59,60}$ ) and gromacs_2016 (ref. ${ }^{61}$ ). Then, the model was put into a $40 \mathrm{~nm}^{3}$ water box. After minimization and equation for $10 \mathrm{~ns}$ at $300 \mathrm{~K}$ using NPT ensemble, we pushed two Fabs together by applying $1 \mathrm{~kJ} \mathrm{~mol}^{-1}$ force on the binding site of digoxin at a rate of $0.1 \mathrm{~nm}$ per $1000 \mathrm{ps}$. The distance of the binding site was varied from 14 to $5 \mathrm{~nm}$. Then, $10 \mathrm{~ns}$ equation simulation was carried out to remove unrealistic conflict. The final distance between binding sites of two Fabs was $5.4 \mathrm{~nm}$. The binding site is constituted by residues: A_Ser34, A_Gln89, A_Gly91, A_Tyr94, B_Tyr35, B_His95, B_Gly97, B_Tyr100, and corresponding residues in chain $\mathrm{C}, \mathrm{D}$. The distances between two binding sites was counted by measuring the mass centers of the two groups. In all simulation, a position restraint of $1000 \mathrm{~kJ} \mathrm{~mol}^{-1}$ was applied on backbone beam of $\mathrm{Fc}_{\mathrm{c}}$ part. To hold the conformation stability of Fabs, elastic network (between beams with distance in the range of $0.5-2 \mathrm{~nm}$ ) with a force of $500 \mathrm{~kJ} \mathrm{~mol}^{-1}$ was applied. Moreover, to insure the flexibility of linker parts (residue B230-B250, D230-D250) between Fc and Fabs, no restraint was implemented in linker parts.

Umbrella sampling. Umbrella sampling is a method that calculates free energy along a reaction coordinate based on MD simulation sampling. We used weighted histogram analysis method (WHAM) to estimate the PMF of distance changing of Fabs. Pulling was initialed with the conformations that have a distance of $5.4 \mathrm{~nm}$ from two binding sites. The pulling force was set to $1 \mathrm{~kJ} \mathrm{~mol}^{-1}$ and pulling rate was set to $0.01 \mathrm{~nm}$ per $100 \mathrm{ps}$. The distance was altered from 5.4 to $17.3 \mathrm{~nm}$. Snapshot of every $0.1 \mathrm{~nm}$ was fetched to perform $1 \mathrm{~ns}$ harmonic constraint MD simulation A harmonic potential, $f(x)=-k x^{2}\left(k=100 \mathrm{~kJ} \mathrm{~mol}^{-1} \mathrm{~nm}^{-2}\right)$, was applied. The $12.0 \mathrm{~nm}$ distance was evenly divided into 120 windows. The conformation sampled of each window was used for PMF calculations with WHAM. The result was presented in Fig. 4b.

Reporting summary. Further information on research design is available in the Nature Research Reporting Summary linked to this article.

\section{Data availability}

The authors declare that the main data supporting the findings of this study are available within the article and Supplementary Information files. Extra data are available from the corresponding author upon request. The source data underlying Figs. 1e, $2 c, 3 c$, d, and 4a, b and Supplementary Figs. 1d, 3b, 6d, 18b,d, 19a-c, 20a, b, 24b, 28b, and 32b are provided as a Source Data file. Source data are provided with this paper. 
Received: 15 January 2020; Accepted: 26 May 2020;

Published online: 19 June 2020

\section{References}

1. North, J. R. Immunosensors: antibody-based biosensors. Trends Biotechnol. 3, 180-186 (1985).

2. Nam, J. M., Thaxton, C. S. \& Mirkin, C. A. Nanoparticle-based bio-bar codes for the ultrasensitive detection of proteins. Science 301, 1884-1886 (2003).

3. Sliwkowski, M. X. \& Mellman, I. Antibody therapeutics in cancer. Science 341, 1192-1198 (2013).

4. Bosch, X., Saiz, A. \& Ramos-Casals, M. The BIOGEAS Study Group. Monoclonal antibody therapy-associated neurological disorders. Nat. Rev. Neurol. 7, 165-172 (2011).

5. Burton, D. R. Antibodies, viruses and vaccines. Nat. Rev. Immunol. 2, 706-713 (2002).

6. Andrews, M. C. \& Wargo, J. A. Cancer evolution during immunotherapy. Cell 171, 740-742 (2017).

7. Gautam, S. et al. The transcription factor c-Myb regulates $\mathrm{CD} 8^{+} \mathrm{T}$ cell stemness and antitumor immunity. Nat. Immunol. 20, 337-349 (2019).

8. Gimzewski, J. K. \& Joachim, C. Nanoscale science of single molecules using local probes. Science 283, 1683-1688 (1999).

9. Rini, J. M., Schulzegahmen, U. \& Wilson, I. A. Structural evidence for induced fit as a mechanism for antibody-antigen recognition. Science 255, 959-965 (1992).

10. Wlodarski, T. \& Zagrovic, B. Conformational selection and induced fit mechanism underlie specificity in noncovalent interactions with ubiquitin. Proc. Natl. Acad. Sci. USA 106, 19346-19351 (2009).

11. Harris, L. J., Larson, S. B., Skaletsky, E. \& McPherson, A. Comparison of the conformations of two intact monoclonal antibodies with hinges. Immunol. Rev. 163, 35-43 (1998).

12. Saphire, E. O. et al. Crystal structure of a neutralizing human IgG against HIV-1: a template for vaccine design. Science 293, 1155-1159 (2001).

13. He, Y. \& Bjorkman, P. J. Structure of FcRY, an avian immunoglobulin receptor related to mammalian mannose receptors, and its complex with $\operatorname{IgY}$. Proc. Natl. Acad. Sci. USA 108, 12431-12436 (2011).

14. Jensen, R. K. et al. Structure of intact IgE and the mechanism of ligelizumab revealed by electron microscopy. Allergy https://doi.org/10.1111/all.14222 (2020).

15. Alsteens, D. et al. Atomic force microscopy-based characterization and design of biointerfaces. Nat. Rev. Mater. 2, 17008 (2017).

16. Benaglia, S. et al. Fast and high-resolution mapping of elastic properties of biomolecules and polymers with bimodal AFM. Nat. Protoc. 13, 2890-2907 (2018).

17. Kodera, N., Yamamoto, D., Ishikawa, R. \& Ando, T. Video imaging of walking myosin V by high-speed atomic force microscopy. Nature 468, 72-76 (2010).

18. Uchihashi, T., Iino, R., Ando, T. \& Noji, H. High-speed atomic force microscopy reveals rotary catalysis of rotorless F(1)-ATPase. Science 333, 755-758 (2011).

19. Dufrene, Y. F. et al. Imaging modes of atomic force microscopy for application in molecular and cell biology. Nat. Nanotechnol. 12, 295-307 (2017).

20. San Paulo, A. \& Garcia, R. High-resolution imaging of antibodies by tappingmode atomic force microscopy: attractive and repulsive tip-sample interaction regimes. Biophys. J. 78, 1599-1605 (2000).

21. Martinez, N. F. et al. Bimodal atomic force microscopy imaging of isolated antibodies in air and liquids. Nanotechnology 19, 384011 (2008).

22. Makky, A. et al. Substructures high resolution imaging of individual $\operatorname{IgG}$ and IgM antibodies with piezoelectric tuning fork atomic force microscopy. Sens. Actuat. B Chem. 162, 269-277 (2012).

23. Iijima, M. et al. Nano-visualization of oriented-immobilized IgGs on immunosensors by high-speed atomic force microscopy. Sci. Rep. UK 2, 790 (2012).

24. Preiner, J. et al. IgGs are made for walking on bacterial and viral surfaces. Nat. Commun. 5, 4394 (2014).

25. Kienberger, F., Mueller, H., Pastushenko, V. \& Hinterdorfer, P. Following single antibody binding to purple membranes in real time. Embo. Rep. 5, 579-583 (2004).

26. Ido, S. et al. Immunoactive two-dimensional self-assembly of monoclonal antibodies in aqueous solution revealed by atomic force microscopy. Nat. Mater. 13, 265-271 (2014).

27. Hinterdorfer, P. et al. Detection and localization of individual antibodyantigen recognition events by atomic force microscopy. Proc. Natl. Acad. Sci. USA 93, 3477-3481 (1996).

28. Liu, H., Schittny, V. \& Nash, M. A. Removal of a conserved disulfide bond does not compromise mechanical stability of a VHH antibody complex. Nano Lett. 19, 5524-5529 (2019)
29. Schwesinger, F. et al. Unbinding forces of single antibody-antigen complexes correlate with their thermal dissociation rates. Proc. Natl. Acad. Sci. USA 97, 9972-9977 (2000)

30. Strasser, J. et al. Unraveling the macromolecular pathways of IgG oligomerization and complement activation on antigenic surfaces. Nano Lett. 19, 4787-4796 (2019).

31. Strasser, J. et al. Weak fragment crystallizable $(\mathrm{Fc})$ domain interactions drive the dynamic assembly of IgG oligomers upon antigen recognition. ACS Nano 14, 2739-2750 (2019).

32. Klein, J. S. \& Bjorkman, P. J. Few and far between: how HIV may be evading antibody avidity. PLoS Pathog. 6, e1000908 (2010).

33. Pluckthun, A. HIV antibodies with a split personality. Nature 467, 537-538 (2010).

34. Rothemund, P. W. Folding DNA to create nanoscale shapes and patterns. Nature 440, 297-302 (2006)

35. Ge, Z., Gu, H., Li, Q. \& Fan, C. Concept and development of framework nucleic acids. J. Am. Chem. Soc. 140, 17808-17819 (2018).

36. Ding, B. Q. et al. Gold nanoparticle self-similar chain structure organized by DNA origami. J. Am. Chem. Soc. 132, 3248-3249 (2010).

37. Liu, X. et al. Complex silica composite nanomaterials templated with DNA origami. Nature 559, 593-598 (2018).

38. Rinker, S. et al. Self-assembled DNA nanostructures for distance-dependent multivalent ligand-protein binding. Nat. Nanotechnol. 3, 418-422 (2008).

39. Mao, X. H. et al. Directing curli polymerization with DNA origami nucleators. Nat. Commun. 10, 1395 (2019).

40. Voigt, N. V. et al. Single-molecule chemical reactions on DNA origami. Nat. Nanotechnol. 5, 200-203 (2010).

41. Shaw, A. et al. Binding to nanopatterned antigens is dominated by the spatial tolerance of antibodies. Nat. Nanotechnol. 14, 184-190 (2019).

42. Tolar, P. Great stretches for your antibody workout. Nat. Nanotechnol. 14 101-102 (2019).

43. Fu, J. L. et al. Multi-enzyme complexes on DNA scaffolds capable of substrate channelling with an artificial swinging arm. Nat. Nanotechnol. 9, 531-536 (2014).

44. Hu, Q. Q., Li, H., Wang, L. H., Gu, H. Z. \& Fan, C. H. DNA nanotechnologyenabled drug delivery systems. Chem. Rev. 119, 6459-6506 (2019).

45. Zhang, X. et al. 3D structural fluctuation of IgG1 antibody revealed by individual particle electron tomography. Sci. Rep. UK 6, 09803 (2014).

46. Bongini, L. et al. Freezing immunoglobulins to see them move. Proc. Natl. Acad. Sci. USA 101, 16466-16471 (2004).

47. Diebolder, C. A. et al. Complement is activated by IgG hexamers assembled at the cell surface. Science 343, 1260-1263 (2014).

48. Saphire, E. O. et al. Crystal structure of a neutralizing human IgG against HIV-1: a template for vaccine design. Science 293, 1155-1159 (2001).

49. Smith, T. J. et al. Structure of a human rhinovirus bivalently bound antibody complex-implications for viral neutralization and antibody flexibility. Proc. Natl. Acad. Sci. USA 90, 7015-7018 (1993).

50. Sandin, S. et al. Structure and flexibility of individual immunoglobulin G molecules in solution. Structure 12, 409-415 (2004).

51. Klein, J. S. et al. Examination of the contributions of size and avidity to the neutralization mechanisms of the anti-HIV antibodies b12 and 4E10. Proc Natl. Acad. Sci. USA 106, 7385-7390 (2009).

52. Müller, K. M., Arndt, K. M. \& Plückthun, A. Model and simulation of multivalent binding to fixed ligands. Anal. Biochem. 261, 149-158 (1998).

53. Bongini, L. et al. A dynamical study of antibody-antigen encounter reactions. Phys. Biol. 4, 172-180 (2007)

54. Acimovic, S. S. et al. Antibody-antigen interaction dynamics revealed by analysis of single-molecule equilibrium fluctuations on individual plasmonic nanoparticle biosensors. ACS Nano 12, 9958-9965 (2018).

55. Beuwer, M. A., Prins, M. W. \& Zijlstra, P. Stochastic protein interactions monitored by hundreds of single-molecule plasmonic biosensors. Nano Lett. 15, 3507-3511 (2015).

56. De Michele, C., De Los Rios, P., Foffi, G. \& Piazza, F. Simulation and theory of antibody binding to crowded antigen-covered surface. PLoS Comput. Biol. 12, 1004752 (2016).

57. $\mathrm{Wu}, \mathrm{N}$. et al. In situ monitoring of single molecule binding reactions with time-lapse atomic force microscopy on functionalized DNA origami. Nanoscale 3, 2481-2484 (2011).

58. Macdonald, R. C. et al. Small-volume extrusion apparatus for preparation of large, unilamellar vesicles. Biochim. Biophys. Acta 1061, 297-303 (1991)

59. Monticelli, L. et al. The MARTINI coarse grained force field: extension to proteins. J. Chem. Theory Comput. 4, 819-834 (2008).

60. Marrink, S. J. et al. The MARTINI force field: coarse grained model for biomolecular simulations. J. Phys. Chem. B 111, 7812-7824 (2007).

61. Van Der Spoel, D. et al. GROMACS: fast, flexible, and free. J. Comput. Chem. 26, 1701-1718 (2005) 


\section{Acknowledgements}

This work was supported by the National Key Research and Development Program (2016YFA0201200, 2018YFA0902600), the National Natural Science Foundation of China (31670871, 21834007, 21991134, and 21603262), the Key Research Program of Frontier Sciences, CAS (QYZDJ-SSW-SLH019), the Open Large Infrastructure Research, and the LU JIAXI International team program supported by CAS, the Innovative research team of high-level local universities in Shanghai, and the K.C. Wong Foundation at Shanghai Jiao Tong University.

\section{Author contributions}

C.F., B.L., and J.H. conceived and supervised the study. P.Z. and X.L. designed and performed experiments. H.A. and T.A. helped HS-AFM experiments. P.L., X.L., and J.L. performed MD simulation. F.W. and L.W. performed TIRF experiments and analyzed the data. X.L., P.Z., B.L., and C.F. wrote the paper. All authors analyzed data and contributed to writing the manuscript.

\section{Competing interests}

The authors declare no competing interests.

\section{Additional information}

Supplementary information is available for this paper at https://doi.org/10.1038/s41467020-16949-4.
Correspondence and requests for materials should be addressed to J.H., B.L. or C.F.

Peer review information Nature Communications thanks Alice Pyne and the other anonymous reviewer(s) for their contribution to the peer review of this work.

Reprints and permission information is available at http://www.nature.com/reprints

Publisher's note Springer Nature remains neutral with regard to jurisdictional claims in published maps and institutional affiliations.

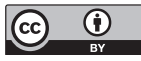

Open Access This article is licensed under a Creative Commons Attribution 4.0 International License, which permits use, sharing, adaptation, distribution and reproduction in any medium or format, as long as you give appropriate credit to the original author(s) and the source, provide a link to the Creative Commons license, and indicate if changes were made. The images or other third party material in this article are included in the article's Creative Commons license, unless indicated otherwise in a credit line to the material. If material is not included in the article's Creative Commons license and your intended use is not permitted by statutory regulation or exceeds the permitted use, you will need to obtain permission directly from the copyright holder. To view a copy of this license, visit http://creativecommons.org/licenses/by/4.0/.

(C) The Author(s) 2020 